\title{
Boson Fields Under a General Class of Local Relativistic Invariant Interactions
}

\author{
RAPHAEL HøEGH-KROHN \\ Mathematical Institute, Oslo University, Norway
}

Received May 27, 1969

\begin{abstract}
We consider a boson field $\varphi(x)$ under an interaction of the form $\int_{R^{3}} V(\varphi(x)) d x$, where $V(\alpha)$ is a bounded continuous real function of a real variable $\alpha$. If $V(\alpha)$ has a uniformly continuous and bounded first derivative, we prove that the Heisenberg picture field exists as weak limits of the Heisenberg picture fields corresponding to the cut-off interaction.
\end{abstract}

\section{Introduction}

The object of this paper is to study a general class of quantum fields with a local relativistic invariant interaction in four space time dimensions. The fields will be self interacting boson fields, with energy operator of the form

$$
H=H_{0}+\int_{R^{3}} V(\varphi(x)) d x .
$$

$H_{0}$ is the free energy operator of a free boson field $\varphi(x)$ of strictly positive mass $m . V(\alpha)$ is a real function of a real variable $\alpha$, such that $V(\alpha)$ is bounded, continuous and with a bounded and uniformly continuous first derivative.

In two space time dimensions Glimm [1] has investigated the case where $V(\alpha)$ is a polynomial containing only terms of even degree and a positive leading coefficient. For this case he proves that, after renormalization of the interaction by introducing the Wick product, the total energy with a space cut off interaction becomes a semi bounded symmetric operator on the Fock space. The case $V(\alpha)=\lambda \alpha^{4}$ and still in two space time dimensions, can be treated more thoroughly, as shown by Glimm and Jaffé [4]. Glimm was also able to treat the case $V(\alpha)=\lambda \alpha^{4}$ in three space time dimensions [2]. The author's reason for studying interactions given by bounded continuous functions instead of polynomials, is strictly that of mathematical convenience, and he hopes that may be in this way enough experience can be gained, so that later on one may be able to treat more realistic models. 
The advantage from a mathematical point of view in studying interactions coming from bounded functions $V(\alpha)$, was also to some extent demonstrated in a previous paper [6], where the author was able to prove existence of the asymptotic fields for the cut-off interactions.

The idea behind this paper is first to introduce the cut-off interactions, and to study the Heisenberg picture fields for the cut-off interactions. Then we use compactness arguments to prove that the weak limits of these fields exists as the cut-off is removed. The idea of studying the weak limits of Heisenberg picture fields was also used by Glimm to remove the space cut-off for the Yukawa interaction with a momentum cut-off [3].

\section{The Heisenberg Picture Fields}

We shall use the Fock space representation. The Fock space $\mathscr{F}$ is a Hilbert space where the elements are sequences of functions $f=\left\{f_{0}, f_{1}, \ldots\right\}$ with $f_{n}=f_{n}\left(p_{1}, p_{2}, \ldots, p_{n}\right)$ a symmetrical function of $n$ variables $p_{1}, \ldots, p_{n} ; p_{i} \in R^{3}$. The inner product in $\mathscr{F}$ is given by

$$
(f, g)=\sum_{n=0}^{\infty} n ! \int \ldots \int \bar{f}_{n}\left(p_{1}, \ldots, p_{n}\right) g_{n}\left(p_{1}, \ldots, p_{n}\right) \frac{d p_{1}}{\omega\left(p_{1}\right)} \ldots \frac{d p_{n}}{\omega\left(p_{n}\right)}
$$

where $\omega(p)=\left(p^{2}+m^{2}\right)^{\frac{1}{2}}$ and $m>0$. The annihilation operator $a(p)$ is defined by

$$
(a(p) f)_{n}\left(p_{1}, \ldots, p_{n}\right)=(n+1) \omega(p)^{-\frac{1}{2}} f_{n+1}\left(p, p_{1}, \ldots, p_{n}\right) .
$$

The creation operator $a^{*}(p)$ is the formal adjoint of $a(p)$, and we have

$$
\left[a(p), a^{*}\left(p^{\prime}\right)\right]=\delta\left(p-p^{\prime}\right) .
$$

The free energy operator $H_{0}$ is defined by

$$
\left(H_{0} f\right)_{n}\left(p_{1}, \ldots, p_{n}\right)=\sum_{i=1}^{n} \omega\left(p_{i}\right) f_{n}\left(p_{1}, \ldots, p_{n}\right) .
$$

$H_{0}$ is obviously self adjoint on its natural domain of definition $D_{0}$.

For $h \in L_{2}\left(R^{3}\right)$, it is well known that $a(h)=\int a(p) h(p) d p$ and $a^{*}(h)=\int a^{*}(p) h(p) d p$ are closed operators with domains containing $D_{0}$, and that $a^{*}(\bar{h})$ is the adjoint of $a(h)$. Moreover $a^{*}(h)+a(\bar{h})$ is a self adjoint operator that is essentially self adjoint on $D_{0}$.

The field operators $\varphi(x)$ are given in terms of the annihilationcreation operators by

$$
\varphi(x)=2^{-\frac{1}{2}}(2 \pi)^{-\frac{3}{2}} \int_{R^{3}}\left(e^{i p x} a(p)+e^{-i p x} a^{*}(p)\right) \omega(p)^{-\frac{1}{2}} d p .
$$


$\varphi(x)$ is of course just as $a(p)$ and $a^{*}(p)$ an improper operator function, or if we want an operator valued distribution; and only when $\varphi(x)$ is integrated against sufficiently smooth test functions, do we get operators. For real $h$ we set

$$
\varphi(h)=\int_{R^{3}} \varphi(x) h(x) d x .
$$

From what is said above, about the annihilation-creation operators, we see that if $\hat{h} \omega^{-\frac{1}{2}}$ is in $L_{2}$, where $\hat{h}$ is the Fourier transform of $h$, then $\varphi(h)$ is a self adjoint operator which is essentially self adjoint on $D_{0}$.

Let $g$ be in $C_{0}^{\infty}\left(R^{3}\right)$, such that $g \geqq 0, g(x)=g(-x), \int g(x) d x=1$ and $g$ has support in the open sphere of radius 1 and center at the origin in $R^{3}$. Set $g_{\varepsilon}(x)=\varepsilon^{-1} g\left(\frac{x}{\varepsilon}\right)$, then $g_{\varepsilon}$ has support in the sphere of radius $\varepsilon$, and $g_{\varepsilon}$ tends to Dirac's $\delta$-distribution as $\varepsilon$ tends to zero. The cut-off field operators are defined by

$$
\varphi_{\varepsilon}(x)=\int g_{\varepsilon}(x-y) \varphi(y) d y .
$$

By what is said above about the field operators we see that $\varphi_{\varepsilon}(x)$ are self adjoint operators which are all essentially self adjoint on $D_{0}$.

Let $V(\alpha)$ be a bounded continuous real function. Then $V\left(\varphi_{\varepsilon}(x)\right)$ is a bounded self adjoint operator, such that $\left\|V\left(\varphi_{\varepsilon}(x)\right)\right\| \leqq\|V\|_{\infty}=\sup _{\alpha}|V(\alpha)|$.

Since

$$
V\left(\varphi_{\varepsilon}(x)\right)=U(-x) V\left(\varphi_{\varepsilon}(0)\right) U(x),
$$

where $U(x)$ is the strongly continuous unitary group defined by

$$
(U(x) f)_{n}\left(p_{1}, \ldots, p_{n}\right)=e^{i \sum_{n=1}^{n} x p_{j}} f_{n}\left(p_{1}, \ldots, p_{n}\right),
$$

we see that $V\left(\varphi_{\varepsilon}(x)\right)$ is strongly continuous in $x$.

We now define the cut-off interaction energy by

$$
V_{\varepsilon, r}=\int_{|x| \leqq r} V\left(\varphi_{\varepsilon}(x)\right) d x
$$

where the integral is a strong integral. We see that $V_{\varepsilon, r}$ is a bounded self adjoint operator, and we have the following $\varepsilon$-independent estimate for its norm

$$
\left\|V_{\varepsilon, r}\right\| \leqq \frac{4 \pi}{3} r^{3}\|V\|_{\infty} .
$$

The cut-off energy operator is defined by

$$
H_{\varepsilon, r}=H_{0}+V_{\varepsilon, r} .
$$


Since $V_{\varepsilon, r}$ is a bounded self adjoint operator we get that $H_{\varepsilon, r}$ is a self adjoint operator with the same domain $D_{0}$ as $H_{0}$.

Let $h$ be real and in $L_{2}$, we then define

$$
\begin{aligned}
\varphi^{t}(h) & =e^{-i t H_{0}} \varphi(h) e^{i t H_{0}}, \\
\varphi_{\varepsilon}^{t}(x) & =e^{-i t H_{0}} \varphi_{\varepsilon}(x) e^{i t H_{0}}, \\
\varphi_{\varepsilon, r, t}(h) & =e^{-i t H_{\varepsilon}, r} \varphi(h) e^{i t H_{\varepsilon}, r} .
\end{aligned}
$$

We see that all operators defined above are self adjoint operators with domain containing $D_{0}$.

We define also

$$
V_{\varepsilon, r}(t)=e^{-i t H_{0}} V_{\varepsilon, r} e^{i t H_{0}} .
$$

It is well known that the product $\varphi^{t_{1}}\left(h_{1}\right) \varphi^{t_{2}}\left(h_{2}\right)$ is defined on the domain of $H_{0}$ and that on this domain

$$
\left[\varphi^{t_{1}}\left(h_{1}\right), \varphi^{t_{2}}\left(h_{2}\right)\right]=\iint d x d y \Delta\left(x-y, t_{1}-t_{2}\right) h_{1}(x) h_{2}(y)
$$

where

$\Delta(x, t)=-i \varepsilon(t)\left[\frac{1}{2 \pi} \delta\left(t^{2}-x^{2}\right)-\frac{m}{4 \pi} \theta\left(t^{2}-x^{2}\right) \cdot\left(t^{2}-x^{2}\right)^{-\frac{1}{2}} J_{1}\left(m\left(t^{2}-x^{2}\right)^{\frac{1}{2}}\right)\right]$

for $t \neq 0$ and $\Delta(x, 0)=0$.

From now on.we shall assume that $V(\alpha)$ has a bounded uniformly continuous derivative $V^{\prime}(\alpha)$.

Lemma 1. Let $h$ be in $L_{2}$ and assume that $V^{\prime}(\alpha)$ is bounded and uniformly continuous. Then $V_{\varepsilon, r}(s)$ leaves the domain of $\varphi^{t}(h)$ invariant, the commutator of these two operators is a bounded operator given by

$$
\left[\varphi^{t}(h), V_{\varepsilon, r}(s)\right]=\int_{|x| \leqq r} d x \iint d z d y h(y) g_{\varepsilon}(x-z) \Delta(y-z, t-s) V^{\prime}\left(\varphi_{\varepsilon}^{s}(x)\right) .
$$

Proof. We start with proving that $V_{\varepsilon, r}(s)$ leaves the domain of $\varphi^{t}(h)$ invariant. Let $\psi$ be in the domain of $\varphi^{t}(h)$, then we shall prove that $e^{i \tau \varphi^{t}(h)} V_{\varepsilon, r}(s) \psi$ is strongly differentiable with respect to $\tau$, and hence $V_{\varepsilon, r}(s) \psi$ is in the domain of $\varphi^{t}(h)$.

$$
e^{i \tau \varphi^{t}(h)} V_{\varepsilon, r}(s) \psi=e^{i \tau \varphi^{t}(h)} V_{\varepsilon, r}(s) e^{-i \tau \varphi^{t}(h)} e^{i \tau \varphi^{t}(h)} \psi .
$$

But $e^{i \tau \varphi^{t}(h)} \psi$ is strongly differentiable with respect to $\tau$, hence it is enough

$$
e^{i \tau \varphi^{t}(h)} V_{\varepsilon, r}(s) e^{-i \tau \varphi^{t}(h)}=\int_{|x| \leqq r} d x V\left(e^{i \tau \varphi^{t}(h)} \varphi_{\varepsilon}^{s}(x) e^{-i \tau \varphi^{t}(h)}\right)
$$

is strongly differentiable with respect to $\tau$.

Using (2.2) we get that this is equal to

$$
\int_{|x| \leqq r} V\left(\varphi_{\varepsilon}^{s}(x)+i \tau \iint d y d z h(y) g_{\varepsilon}(x-z) \Delta(y-z, t-s)\right) .
$$


Since $V(\alpha)$ has a bounded uniformly continuous derivative $V^{\prime}(\alpha)$, we have that $\frac{1}{\tau}(V(\alpha+c \tau)-V(\alpha))$ tends to $c V^{\prime}(\alpha)$ uniformly in $\alpha$. Hence for any self adjoint operator $A, \frac{1}{\tau}(V(A+c \tau)-V(A))$ tends to $c V^{\prime}(A)$ in norm, and the convergence is uniform in $A$ where $A$ range over the set of all self adjoint operators. Hence

$$
V\left(\varphi_{\varepsilon}(x)+i \tau \iint d y d z h(y) g_{\varepsilon}(x-z) \Delta(y-z, t-s)\right)
$$

is norm differentiable in $\tau$ and the convergence of the difference to the derivative is uniform in $x$. Hence

$$
\int_{|x| \leqq r} V\left(\varphi_{\varepsilon}(x)+i \tau \iint d y d z h(y) g_{\varepsilon}(x-z) \Delta(y-z, t-s)\right)
$$

is norm differentiable with respect to $\tau$, and with derivative given by

$$
i \int_{|x| \leqq r} d x \iint d y d z h(y) g_{\varepsilon}(x-z) \Delta(y-z, t-s) V^{\prime}\left(\varphi_{\varepsilon}^{s}(x)\right) .
$$

This proves that $V_{\varepsilon, r}(s) \psi$ is in the domain of $\varphi^{t}(h)$. By differentiating the first formula of this proof we see that

$$
\begin{aligned}
\varphi^{t}(h) V_{\varepsilon, r}(s) \psi= & V_{\varepsilon, r}(s) \varphi^{t}(h) \psi \\
& \quad+\int_{|x| \leqq r} d x \iint d y d z h(y) g_{\varepsilon}(x-z) \Delta(y-z, t-s) V^{\prime}\left(\varphi_{\varepsilon}^{s}(x)\right) .
\end{aligned}
$$

This gives us the formula for the commutator as given in the lemma. Using this formula together with the assumption that $V^{\prime}(\alpha)$ is bounded, we get that the commutator is bounded. This proves the lemma.

Corollary 1. $\left\|\left[\varphi^{t}(h), V_{\varepsilon, r}(s)\right]\right\| \leqq C|t-s|^{2}\left\|V^{\prime}\right\|_{\infty}\|h\|_{1}$ where $C$ depends only on the mass $m$ of the free field.

Proof. From Lemma 1 we get that

$$
\left\|\left[\varphi^{t}(h), V_{\varepsilon, r}(s)\right]\right\| \leqq\left\|V^{\prime}\right\|_{\infty} \int d z\left|\int h(y) \Delta(y-z, t-s)\right|
$$

and the estimate in the corollary follows then from (2.3) and the asymptotic behaviour of $J_{1}$. This proves the corollary.

The Heisenberg picture fields corresponding to the cut-off interaction is given by $\varphi_{\varepsilon, r, t}(h)$. We intend to use the fact that the estimate of Corollary 1 is uniform in $\varepsilon$ to select a sequence $\varepsilon_{n}$ tending to zero such that $\varphi_{\varepsilon_{n}, r, t}(h)$ converge weakly for all $t$ and $r$, and all $h$ in $L_{2} \cap L_{1}$. The way we do this is by showing that Corollary 1 implies that $\varphi_{\varepsilon, r, t}(h)$ is equicontinuous as functions of $r, t$ and $h$ with respect to $\varepsilon$. Then we use the Ascoli theorem to pick out the sequence $\varepsilon_{n}$.

Let $\psi$ be in $D_{0}$. Since $D_{0}$ is the domain of $H_{0}$ as well as $H_{\varepsilon, r}$, and also therefore $e^{i s H_{\varepsilon, r}}$ leaves $D_{0}$ invariant, we get that $e^{-i(s-t) H_{0}} e^{i s H_{\varepsilon, r}} \psi$ 
is strongly differentiable with respect to $s$, and the strong derivative is given by

$$
e^{-i(s-t) H_{0}} i V_{\varepsilon, r} e^{i s H_{\varepsilon, r}} \psi=i V_{\varepsilon, r}(s-t) e^{-i(s-t) H_{0}} e^{i s H_{\varepsilon, r}} \psi .
$$

Let $h$ be in $L_{2}$. Since $D_{0}$ is contained in the domain of $\varphi(h)$, we get from Lemma 1 that the derivative is in the domain of $\varphi(h)$. Using now that $\varphi(h)$ is closed we see that

$$
\frac{d}{d s} \varphi(h) e^{-i(s-t) H_{0}} e^{i s H_{\varepsilon, r}} \psi=\varphi(h) i V_{\varepsilon, r}(s-t) e^{-i(s-t) H_{0}} e^{i s H_{\varepsilon, r}} \psi .
$$

where the derivative is taken in the strong sense. From this we get that if $\psi_{1}$ and $\psi_{2}$ are in $D_{0}$ then

$$
\begin{aligned}
\left(\psi_{1}, e^{-i s H_{\varepsilon, r}} e^{i(s-t) H_{0}} \varphi(h) e^{-i(s-t) H_{0}} e^{i s H_{\varepsilon, r}} \psi_{2}\right) & \\
& =\left(e^{-i(s-t) H_{0}} e^{i s H_{\varepsilon, r}} \psi_{1}, \varphi(h) e^{-i(s-t) H_{0}} e^{i s H_{\varepsilon, r}} \psi_{2}\right)
\end{aligned}
$$

is differentiable with respect to $s$ and the derivative is given by

$$
\begin{aligned}
\left(e^{-i(s-t) H_{0}} i V_{\varepsilon, r} e^{i s H_{\varepsilon, r}}\right. & \left.\psi_{1}, \varphi(h) e^{-i(s-t) H_{0}} e^{i s H_{\varepsilon, r}} \psi_{2}\right) \\
& +\left(e^{-i(s-t) H_{0}} e^{i s H_{\varepsilon, r}} \psi_{1}, \varphi(h) e^{-i(s-t) H_{0}} i V_{\varepsilon, r} e^{i s H_{\varepsilon, r}} \psi_{2}\right) \\
= & \left(\psi_{1}, e^{-i s H_{\varepsilon, r}}\left[\varphi^{t-s}(h), i V_{\varepsilon, r}\right] e^{i s H_{\varepsilon, r}} \psi_{2}\right) .
\end{aligned}
$$

By integrating the derivative we then get

$$
\left(\psi_{1},\left(\varphi_{\varepsilon, r, t}(h)-\varphi^{t}(h)\right) \psi_{2}\right)=\int_{0}^{t} d s\left(\psi_{1}, e^{-i s H_{\varepsilon, r}}\left[\varphi^{t-s}(h), i V_{\varepsilon, r}\right] e^{i s H_{\varepsilon, r}} \psi_{2}\right)
$$

Since the right hand side is a bounded operator by Lemma 1 and $D_{0}$ is dense in $\mathscr{F}$ we get

$$
\varphi_{\varepsilon, r, t}(h)-\varphi^{t}(h)=\int_{0}^{t} d s e^{-i s H_{\varepsilon, r}}\left[\varphi^{t-s}(h), i V_{\varepsilon, r}\right] e^{i s H_{\varepsilon, r}},
$$

where the integral on the right hand side is a weak integral.

Lemma 2. Let $h$ be in $L_{1} \cap L_{2}$, then

$$
\left[\varphi^{t}(h), i V_{\varepsilon, r}(s)\right]
$$

is norm equicontinuous in $t$ with respect to $\varepsilon$ and $r$.

Proof. Let $h$ be in $C_{0}^{\infty}$. Then we get from Lemma 1 that

$$
\begin{aligned}
\left\|\left[\varphi^{t_{1}}(h), i V_{\varepsilon, r}(s)\right]-\left[\varphi^{t_{2}}(h), i V_{\varepsilon, r}(s)\right]\right\| \\
\leqq\left\|V^{\prime}\right\|_{\infty} \int d z\left|\int h(y)\left(\Delta\left(y-z, t_{1}-s\right)-\Delta\left(y-z, t_{2}-s\right)\right) d y\right|
\end{aligned}
$$

We see that the right hand side is independent of $\varepsilon$ and $r$. Moreover as $t_{1}$ tends to $t_{2} \int h(y) \Delta\left(y-z, t_{1}-s\right) d y$ tends uniformly in $z$ to 
$\int h(y) \Delta\left(y-z, t_{2}-s\right) d y$, and as the functions are zero outside a fixed compact we see that the right hand side tends to zero. Hence we have proved that for $h$ in $C_{0}^{\infty}\left[\varphi^{t}(h), i V_{\varepsilon, r}(s)\right]$ is norm equicontinuous. But $C_{0}^{\infty}$ is dense in $L_{1}$ and by linearity in $h$ and corollary 1 we get norm equicontinuity for all $h$ in $L_{1}$. This proves the lemma.

Lemma 3. Let $h$ be in $L_{1} \cap L_{2}$, then

$$
\varphi_{\varepsilon, r, t}(h)-\varphi^{t}(h)=\int_{0}^{t} d s e^{-i s H_{\varepsilon, r}}\left[\varphi^{t-s}(h), i V_{\varepsilon, r}\right] e^{i s H_{\varepsilon, r}},
$$

where the integral is a strong integral, and the integrand is strongly continuous.

Proof. By Lemma $2\left[\varphi^{t-s}(h), i V_{\varepsilon, r}\right]$ is a norm continuous function of $s$. Hence the integrand is strongly continuous and therefore strongly integrable. But for strongly integrable functions the strong and the weak integral coinside and the lemma is therefore proved by formula 2.4 .

Corollary 2. For $h$ in $L_{1} \cap L_{2}$, we have that

$$
\left\|\varphi_{\varepsilon, r, t}(h)-\varphi^{t}(h)\right\| \leqq C|t|^{3}\left\|V^{\prime}\right\|_{\infty}\|h\|_{1},
$$

where $C$ depends only on the mass $m$ of the free field.

Proof. We use the norm estimate of Corollary 1 to estimate the integrand of Lemma 3. This gives us the estimate of Corollary 2, and this proves the corollary.

Lemma 4. Let $h$ be in $L_{1} \cap L_{2}$, then $\varphi_{\varepsilon, r, t}(h)-\varphi^{t}(h)$ are norm equicontinuous functions of $t$ with respect to $\varepsilon$ and $r$.

Proof. By Lemma 3 we get for $t_{1} \leqq t_{2}$

$$
\begin{aligned}
& \| \varphi_{\varepsilon, r, t_{1}}(h)-\varphi^{t_{1}}(h)-\varphi_{\varepsilon, r, t_{2}}(h)+\varphi^{t_{2}}(h) \| \\
& \leqq \\
& \int_{0}^{t_{1}} d s\left\{\left\|\left[\varphi^{t_{1}-s}(h), V_{\varepsilon, r}\right]-\left[\varphi^{t_{2}-s}(h), V_{\varepsilon, r}\right]\right\|\right\} \\
& \quad+\int_{t_{1}}^{t_{2}} d s\left\|\left[\varphi^{t_{2}-s}(h), V_{\varepsilon, r}\right]\right\| .
\end{aligned}
$$

By Corollary 1 the integrand of the first integral is uniformly bounded, and by Lemma 2 it tends pointwise to zero as $t_{1}$ tends to $t_{2}$ or $t_{2}$ tends to $t_{1}$, uniformly in $\varepsilon$ and $r$. Hence by Lebesques lemma on dominated convergence the first integral tends to zero as $t_{1}$, tends to $t_{2}$, or $t_{2}$ tends to $t_{1}$, and the convergence is uniform in $\varepsilon$ and $r$. By Corollary 1 the second integral is dominated by $C\left|t_{1}-t_{2}\right| t_{2}^{2}\left\|V^{\prime}\right\|_{\infty}\|h\|_{1}$ which obviously tends to zero uniformly in $\varepsilon$ and $r$. This proves the lemma. 
Lemma 5. $e^{i t H_{\varepsilon, r}}$ is norm continuous in $r$, and the normcontinuity in $r$ is uniform in $\varepsilon$ and on compact intervals in $t$. We have the estimate

$$
\left\|e^{i t H_{\varepsilon, r_{1}}}-e^{i t H_{\varepsilon, r_{2}}}\right\| \leqq \frac{4 \pi}{3}\left|r_{1}^{3}-r_{2}^{3}\right||t|\|V\|_{\infty} .
$$

Proof. Since the domain of $H_{\varepsilon, r}$ is equal to $D_{0}$ for all $r$, we have that $e^{i s H_{\varepsilon, r}}$ leaves $D_{0}$ invariant for all $r$, and therefore $e^{i(t-s) H_{\varepsilon, r_{1}}} e^{i s H_{\varepsilon, r_{2}}}$ is strongly differentiable in $s$ on $D_{0}$ with derivative

$$
\frac{d}{d s} e^{i(t-s) H_{\varepsilon, r_{1}}} e^{i s H_{\varepsilon, r_{2}}}=e^{i(t-s) H_{\varepsilon, r_{1}}} i\left(V_{\varepsilon, r_{2}}-V_{\varepsilon, r_{1}}\right) e^{i s H_{\varepsilon, r_{2}}} .
$$

Integrating both sides, we get on $D_{0}$

$$
e^{i t H_{\varepsilon, r_{2}}}-e^{i t H_{\varepsilon, r_{1}}}=\int_{0}^{t} d s e^{i(t-s) H_{\varepsilon, r_{1}}} i\left(V_{\varepsilon, r_{2}}-V_{\varepsilon, r_{1}}\right) e^{i s H_{\varepsilon, r_{2}}}
$$

Since $D_{0}$ is dense in $\mathscr{F}$ the estimate in the lemma follows from a direct estimate of the norm of the integral above. This proves the lemma.

Lemma 6. Let $h$ be in $L_{1} \cap L_{2}$ then $\varphi_{\varepsilon, r, t}(h)-\varphi^{t}(h)$ is norm continuous in $r$, and the normcontinuity in $r$ is uniform in $\varepsilon$ and in ton compact intervals.

Proof. From Lemma 3 we get

$$
\begin{aligned}
& \varphi_{\varepsilon, r_{1}, t}(h)-\varphi_{\varepsilon, r_{2}, t}(h)=i \int_{0}^{t} d s e^{-i s H_{\varepsilon, r_{1}}}\left[\varphi^{t-s}(h), V_{\varepsilon, r_{1}}\right] e^{i s H_{\varepsilon, r_{1}}}
\end{aligned}
$$

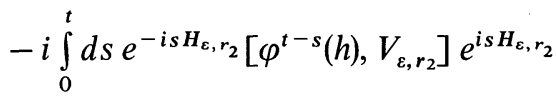

$$
\begin{aligned}
& =i \int_{0}^{t} d s\left\{\left(e^{-i s H_{\varepsilon, r_{1}}}-e^{-i s H_{\varepsilon, r_{2}}}\right)\left[\varphi^{t-s}(h), V_{\varepsilon, r_{1}}\right] e^{i s H_{\varepsilon, r_{1}}}\right. \\
& +e^{-i s H_{\varepsilon, r_{2}}}\left[\varphi^{t-s}(h), V_{\varepsilon, r_{1}}-V_{\varepsilon, r_{2}}\right] e^{i s H_{\varepsilon, r_{1}}} \\
& \left.+e^{-i s H_{\varepsilon, r_{2}}}\left[\varphi^{t-s}(h), V_{\varepsilon, r_{2}}\right]\left(e^{i s H_{\varepsilon, r_{1}}}-e^{i s H_{\varepsilon, r_{2}}}\right)\right\} .
\end{aligned}
$$

The first and the last term of the integrand is estimated uniformly in $\varepsilon$ and in $t$ on compact intervals by Lemma 5 . The second term in the integrand is estimated by $C\left|r_{1}^{3}-r_{2}^{3}\right|$ where $C$ is independent on $\varepsilon$ and on $t$ if $t$ is on a compact interval, by the formula of Lemma 1 . This proves the lemma.

Theorem 1. There exists a sequence $\varepsilon_{n}$ tending to zero, such that for all $h$ in $L_{1} \cap L_{2}$ and all $t$ and $r, \varphi_{\varepsilon_{n}, r, t}(h)-\varphi^{t}(h)$ converge weakly to a limit $\varphi_{r}(h, t)-\varphi^{t}(h)$. The convergence is uniform for $r$ and $t$ on compact intervals, and the limit is normcontinuous in $r$ and $t$. Relative to the strong $L_{1}$ topology, 
the limit is also normcontinuous in $h$ and

$$
\left\|\varphi_{r}(h, t)-\varphi^{t}(h)\right\| \leqq C|t|^{3}\left\|V^{\prime}\right\|_{\infty}\|h\|_{1},
$$

where $C$ depends only on the mass $m$ of the free field.

Proof. Let $\psi_{1}$ and $\psi_{2}$ be in $\mathscr{F}$ and $h$ be in $L_{1} \cap L_{2} .\left(\psi_{1}, \varphi_{\varepsilon, r, t}(\mathrm{~h})-\varphi^{t}(\mathrm{~h}) \psi_{2}\right)$ is then by Corollary 2 a uniformly bounded family of functions of $t$ and $r$, depending on a parameter $\varepsilon$. By Lemma 4 and Lemma 6 it is also an equicontinuous family of functions of $r$ and $t$. The Ascoli theorem then gives the existence of a sequence $\varepsilon_{n}^{\prime}$, tending to zero, such that the corresponding sequence of functions converge uniformly for $t$ and $r$ on compact intervals. By passing to a subsequence $\varepsilon_{n}$ we get uniform convergence for a countable dense set of $\psi_{1}$ and $\psi_{2}$, and a countable set of $h$ that is dense in $L_{1} \cap L_{2}$ in the strong $L_{1}$ topology. The norm estimate of Corollary 2 then gives us convergence, uniformly for $t$ and $r$ on compact intervals, for all $\psi_{1}$ and $\psi_{2}$ in $\mathscr{F}$ and all $h$ in $L_{1} \cap L_{2}$. This proves the weak convergence. To see that the limit is normcontinuous in $t$, we use Lemma 4 which gives us that for all $\varepsilon>0$ there exists a $\delta>0$ independent of $n$ such that

$$
\left\|\varphi_{\varepsilon_{n}, r, t+\tau}(h)-\varphi^{t+\tau}(h)-\varphi_{\varepsilon_{n}, r, t}(h)+\varphi^{t}(h)\right\| \leqq \varepsilon
$$

as soon as $|\tau|<\delta$. Now we use that the set of operators with norm smaller or equal to $\varepsilon$ is weakly closed to get the same estimate for the limit. This proves normcontinuity in $t$, and normcontinuity in $r$ is proved in the same way by using Lemma 6 . Normcontinuity in $h$ follows from the norm estimate of the theorem. Hence it is enough to prove this estimate. But using again that a closed ball of operators is weakly closed, we see that this estimate follows directly from Corollary 2. This proves the theorem.

Lemma 7. Let $H_{0}$ and $H$ be two self adjoint operators on a Hilbert space, such that $V=H-H_{0}$ is bounded. Let $A$ be a bounded operator and define $A_{t}=e^{-i t H} e^{i t H_{0}} A e^{-i t H_{0}} e^{i t H}$. Then we have for $t \geqq 0$,

$$
A_{t}=\sum_{n=0}^{\infty} i^{n} \int_{t \geqq t_{1} \geqq t_{2} \cdots \geqq t_{n} \geqq 0}\left[\ldots\left[A, V\left(t_{1}\right)\right] \ldots, V\left(t_{n}\right)\right] d t_{1} \ldots d t_{n}
$$

where $V(t)=e^{-i t H_{0}} V(t) e^{i t H_{0}}$. The integrals are strong integrals and the sum is norm convergent.

Proof. Since $V$ is bounded, $H$ and $H_{0}$ have the same domain and $e^{i t H}$ and $e^{i t H_{0}}$ leaves this domain invariant. Let $\psi_{1}$ and $\psi_{2}$ be in this domain, then we see that $\left(\psi_{1}, A_{t} \psi_{2}\right)$ is differentiable with respect to $t$ and

$$
\frac{d}{d t}\left(\psi_{1}, A_{t} \psi_{2}\right)=\left(\psi_{1}, e^{-i t H}\left[e^{i t H_{0}} A e^{-i t H_{0}}, i V\right] e^{i t H} \psi_{2}\right) .
$$


By integration we get that

$$
\left(\psi_{1}, A_{t} \psi_{2}\right)=\left(\psi_{1}, A \psi_{2}\right)+\int_{0}^{t} d s\left(\psi_{1}, e^{-i s H} e^{i s H_{0}}[A, i V(s)] e^{i s H_{0}} e^{i s H} \psi_{2}\right) .
$$

Since the domain of $H_{0}$ is dense and $A_{t}, A$ and $V(t)$ are bounded the identity above holds for all $\psi_{1}$ and all $\psi_{2}$. Hence

$$
A_{t}=A+i \int d s e^{-i s H} e^{i s H_{0}}[A, V(s)] e^{-i s H_{0}} e^{i s H},
$$

where the integral is a weak integral. But we see that the integrand is bounded and strongly continuous, hence strongly integrable, and therefore the weak integral coincides with the strong integral. By iterating the formula above we get the formula of Lemma 5. The norm convergence follows from a direct norm estimate. This proves the lemma.

Lemma 8. Let $h$ be in $L_{1} \cap L_{2}$, then for $t \geqq 0$

$$
\varphi_{\varepsilon, r, t}(h)-\varphi^{t}(h)=\sum_{n=1}^{\infty} i^{n} \int_{t \geqq t_{1} \cdots \geqq t_{n} \geqq 0}\left[\ldots\left[\varphi^{t}(h), V_{\varepsilon, r}\left(t_{1}\right)\right] \ldots, V_{\varepsilon, r}\left(t_{n}\right)\right] d t_{1} \ldots d t_{n}
$$

where the integrals are strong integrals and the sum is normconvergent uniformly in $\varepsilon$.

Proof. The formula of Lemma 3 may be written

$$
\varphi_{\varepsilon, r, t}(h)-\varphi^{t}(h)=i \int d s e^{-i s H_{\varepsilon, r}} e^{i s H_{0}}\left[\varphi^{t}(h), V_{\varepsilon, r}(s)\right] e^{-i s} e^{i s H_{\varepsilon, r}} .
$$

By Corollary $1\left[\varphi^{t}(h), V_{\varepsilon, r}(s)\right]$ is bounded uniformly in $\varepsilon$. Hence we may apply the formula of Lemma 7 to the integrand, and this gives us the formula of Lemma 8. To prove that the normconvergence is uniform in $\varepsilon$, we estimate the norm of the $n$ 'th term in the sum by

$$
C\left\|V^{\prime}\right\|_{\infty}\|h\|_{1} \frac{t^{n+1}}{n !}\left\|V_{\varepsilon, r}\right\|^{n-1}
$$

using Corollary 1. But since

$$
\left\|V_{\varepsilon, r}\right\| \leqq \frac{4 \pi}{3} r^{3}\|V\|_{\infty}
$$

we see that the normconvergence is uniform in $\varepsilon$. This proves the lemma.

Definition. For any subset $S$ of $R^{3}$ we define $S_{t}=S+B_{t}$ where $B_{t}=\{x ;|x| \leqq t\} . S_{t}$ is then the set of points in $R^{3}$ which is causally dependent on $S$ up to the time $t$, or in other words $S_{t}$ is the set of points that can be reached, in a time less or equal to $t$, by a light signal emitted from $S$. We shall also use the expression that to sets $S_{1}$ and $S_{2}$ are causally independent up to the time $t$ if $S_{1, t} \cap S_{2}=0$. 
Let $\Lambda$ be any compact set in $R^{3}$. Then we define

and

$$
V_{\varepsilon, \Lambda}=\int V\left(\varphi_{\varepsilon}(x)\right) d x, H_{\varepsilon, \Lambda}=H_{\varepsilon, \Lambda}
$$

$$
\varphi_{\varepsilon, \Lambda, t}(h)=e^{-i t H_{\varepsilon, \Lambda}} \varphi(h) e^{i t H_{\varepsilon, \Lambda}} .
$$

Theorem 2. Let $h$ be in $L_{1} \cap L_{2}$, then $\varphi_{r}(h, t)-\varphi^{t}(h)$ converge in norm in norm to $\varphi(h, t)-\varphi^{t}(h)$. The convergence is uniform for $t$ on compact intervals, and the limit is norm continuous in $t$. Relative to the strong $L_{1}$ topology, the limit is also normcontinuous in $h$ and

$$
\left\|\varphi(h, t)-\varphi^{t}(h)\right\| \leqq C|t|^{3}\left\|V^{\prime}\right\|_{\infty}\|h\|_{1}
$$

where $C$ depends only on the mass $m$ of the free field.

Proof. We shall assume first that $h$ is in $L_{2}$ and have compact support $S$. It follows from the expression for the function $\Delta$ that $\varphi^{s}\left(h_{1}\right)$ and $\varphi^{t}\left(h_{2}\right)$ commute if the support of $h_{1}$ and the support of $h_{2}$ are causally independent up to the time $|t-s|$. This gives us for any two bounded continuous functions $F_{1}$ and $F_{2}$ that $F_{1}\left(\varphi_{\varepsilon}^{s}(x)\right)$ and $F_{2}\left(\varphi_{\varepsilon}^{t}(y)\right)$ commute if $|x-y| \geqq|t-s|+2 \varepsilon$. From Lemma 1 we get for $t \geqq t_{1} \geqq 0$

$$
\begin{aligned}
{\left[\varphi^{t}(h), V_{\varepsilon, r}\left(t_{1}\right)\right] } & =\left[\varphi^{t}(h), V_{\varepsilon, S_{t-t_{1}+\varepsilon}}\left(t_{1}\right)\right] \\
& =\left[\varphi^{t}(h), V_{\varepsilon, \Lambda}\left(t_{1}\right)\right]
\end{aligned}
$$

if $S_{t-t_{1}+\varepsilon} \subset B_{r}$ and $S_{t-t_{1}+\varepsilon} \subset \Lambda$.

Using now that $F_{1}\left(\varphi_{\varepsilon}^{s}(x)\right)$ and $F_{2}\left(\varphi_{\varepsilon}^{t}(y)\right)$ commute if $|x-y| \geqq|t-s|+2 \varepsilon$, we get for $t \geqq t_{1} \geqq t_{2} \geqq 0$.

$$
\begin{aligned}
{\left[\left[\varphi^{t}(h),\right.\right.} & \left.\left.V_{\varepsilon, r}\left(t_{1}\right)\right], V_{\varepsilon, r}\left(t_{2}\right)\right] \\
& =\left[\left[\varphi^{t}(h), V_{\varepsilon, S_{t-t_{1}+\varepsilon}}\left(t_{1}\right)\right], V_{\varepsilon, S_{t-t_{2}+3_{\varepsilon}}}\left(t_{2}\right)\right] \\
& =\left[\left[\varphi^{t}(h), V_{\varepsilon, \Lambda}\left(t_{1}\right)\right], V_{\varepsilon, \Lambda}\left(t_{2}\right)\right]
\end{aligned}
$$

if $S_{t+3 \varepsilon} \subset B_{r}$ and $S_{t+3 \varepsilon} \subset \Lambda$.

In the same way we get for $t \geqq t_{1} \geqq t_{2} \ldots \geqq t_{n} \geqq 0$

$$
\begin{aligned}
& {\left[\ldots\left[\varphi^{t}(h), V_{\varepsilon, r}\left(t_{1}\right)\right] \ldots, V_{\varepsilon, r}\left(t_{n}\right)\right]} \\
& \quad=\left[\ldots\left[\varphi^{t}(h), V_{\varepsilon, S_{t-t_{1}+\varepsilon}}\left(t_{1}\right)\right] \ldots, V_{\varepsilon, S_{t-t_{n}+(2 n+1) \varepsilon}}\left(t_{n}\right)\right] \\
& \quad=\left[\ldots\left[\varphi^{t}(h), V_{\varepsilon, \Lambda}\left(t_{1}\right)\right] \ldots, V_{\varepsilon, \Lambda}\left(t_{n}\right)\right]
\end{aligned}
$$

if $S_{t+(2 n+1) \varepsilon} \subset B_{r}$ and $S_{t+(2 n+1) \varepsilon} \subset A$.

But this proves that the $n$ first terms in the sum in Lemma 8 is independent of $r$ as soon as $S_{t+(2 n+1) \varepsilon} \subset B_{r}$. Assume now that $S_{t}$ is contained in the interior of $B_{r_{1}}$ and that $r_{1} \leqq r_{2}$. Since the sum in Lemma 8 is normconvergent uniformly in $\varepsilon$ we then get that $\varphi_{\varepsilon_{n}, r_{2}, t}(h)-\varphi_{\varepsilon_{n}, r_{1}, t}(h)$ tends to zero in norm as $\varepsilon_{n}$ tends to zero. This proves that the weak limit $\varphi_{r}(h, t)-\varphi^{t}(h)$ is independent of $r$ as soon as $S_{t}$ is contained in the interior 
of $B_{r}$. This proves the normconvergence uniformly for $t$ on compact intervals, if $h$ has compact support. But since functions with compact support is dense in $L_{1} \cap L_{2}$ relative to the strong topology in $L_{1}$, the estimate of Theorem 1 gives us normconvergence uniformly for $t$ on compact intervals for all $h$ in $L_{1} \cap L_{2}$. That the limit is normcontinuous in $t$ follows from uniform convergence. The estimate of the theorem follows immediately from the estimate of Theorem 1, and this gives also the normcontinuity in $h$. This proves the theorem.

Lemma 9. Let $h$ be in $L_{1} \cap L_{2}$, then for $t \geqq 0$

$\varphi_{\varepsilon, \Lambda, t}(h)-\varphi^{t}(h)=\sum_{n=1}^{\infty} i^{n} \int_{t \geqq t_{1} \cdots \geqq t_{n} \geqq 0}\left[\ldots\left[\varphi^{t}(h), V_{\varepsilon, \Lambda}\left(t_{1}\right)\right] \ldots, V_{\varepsilon, \Lambda}\left(t_{n}\right)\right] d t_{1} \ldots d t_{n}$

where the integrals are strong integrals and the sum is normconvergent uniformly in $\varepsilon$ and uniformly in $\Lambda$, with $|\Lambda|<C$. $|\Lambda|$ is the total volume of $\Lambda$.

Proof. This lemma is proved in the same way as Lemma 8 , and only trivial modifications of the proof of Lemma 8 is needed to prove Lemma 9.

We will now prove two theorems which express that the interaction is local.

Theorem 3. Let $\varepsilon_{n}$ be the sequence of Theorem 1 .

Let $h$ be in $L_{2}$ with compact support $S$. Let $\Lambda$ be any compact such that $S_{t}$ is contained in the interior of $\Lambda$. Then $\varphi_{\varepsilon_{n}, \Lambda, t}(h)-\varphi^{t}(h)$ converge weakly to $\varphi(h, t)-\varphi^{t}(h)$ as $n$ tends to infinity.

Proof. From the proof of Theorem 2 we see that the $n$ first terms in the sums in Lemma 8 and Lemma 9 is the same if $S_{t+(2 n+1) \varepsilon} C A$ and $S_{t+(2 n+1) \varepsilon}$ $C \mathrm{~B}_{r}$. Hence $\varphi_{\varepsilon_{n}, A, t}(h)-\varphi_{\varepsilon_{n}, r, t}(h)$ converge to zero in norm by Lemma 8 and Lemma 9, if $S_{t}$ is contained in the interior of $A$ and of $B_{r}$. But from the proof of Theorem 2 we see that $\varphi_{\varepsilon_{n}, r, t}(h)-\varphi^{t}(h)$ converge weakly to $\varphi(h, t)-\varphi^{t}(h)$. This proves the theorem.

Theorem 4. Let $h_{1}$ and $h_{2}$ be in $L_{2}$ with compact supports $S_{1}$ and $S_{2}$. If $S_{1}$ and $S_{2}$ are causally independent up to time $t$, then $\varphi\left(h_{1}, t\right)$ and $\varphi\left(h_{2}\right)$ commute.

Proof. Since $\varphi^{t}\left(h_{1}\right)$ and $\varphi\left(h_{2}\right)$ commute, it is enough to prove that $\varphi\left(h_{1}, t\right)-\varphi^{t}\left(h_{1}\right)$ and $\varphi\left(h_{2}\right)$ commute. But since the set of operators that commute with $\varphi\left(h_{2}\right)$ is weakly closed it is enough to prove that $\varphi_{\varepsilon, r, t}\left(h_{1}\right)$ $-\varphi^{t}\left(h_{1}\right)$ commutes with $\varphi\left(h_{2}\right)$ if $\varepsilon$ is small and $r$ such that $S_{1, t}$ is contained in the interior of $B_{r}$. Using once more that the commutator is weakly closed and Lemma 8 , we see that it is enough to prove that for $t \geqq t_{1}$ $\geqq \cdots \geqq \mathrm{t}_{n} \geqq 0$

$$
\left[\ldots\left[\varphi^{t}\left(h_{1}\right), V_{\varepsilon, r}\left(t_{1}\right)\right] \ldots, V_{\varepsilon, r}\left(t_{n}\right)\right]
$$


commute with $\varphi\left(h_{2}\right)$ for $\varepsilon$ small enough. But by (2.5) we have that (2.6) is equal to

$$
\left[\ldots\left[\varphi^{t}\left(h_{1}\right), V_{\varepsilon, S_{1, t-t_{1}+\varepsilon}}\left(t_{1}\right)\right] \ldots, V_{\varepsilon, S_{1, t-t_{n}+(2 n+1) \varepsilon}}\left(t_{n}\right)\right] .
$$

Using Lemma 1 we see that $\varphi\left(h_{2}\right)$ commutes with $\left[\varphi^{t}\left(h_{1}\right), V_{\varepsilon, S_{1, t-t_{1}+\varepsilon}}\left(t_{1}\right)\right]$ if $S_{1}$ and $S_{2}$ are causally independent up to time $t+\varepsilon$. For $j=2,3, \ldots, n$ we see that $\varphi\left(h_{2}\right)$ commutes with $V_{\varepsilon, S_{1, t-t_{j}+(2 j+1) \varepsilon}}\left(t_{j}\right)$ if $S_{1}$ and $S_{2}$ are causally independent up to time $t+(2 j+1) \varepsilon$. Therefore $\varphi\left(h_{2}\right)$ commutes with (2.7) if $S_{1}$ and $S_{2}$ are causally independent up to time $t+(2 n+1) \varepsilon$. But since $S_{1}$ and $S_{2}$ are compacts, we see that if they are causally independent up to a time $t$, then there exists $\delta>0$ such that $S_{1}$ and $S_{2}$ are causally independent up to time $t+\delta$. Hence for $(2 n+1) \varepsilon<\delta$ we find that $\varphi\left(h_{2}\right)$ commutes with (2.7). This proves the theorem.

For the free Heisenberg picture fields $\varphi^{t}(h)$ we have the following translation invariance

$$
U(-x) \varphi^{t}(h) U(x)=\varphi^{t}\left(h_{x}\right),
$$

where $U(x)$ is the unitary group of space translations introduced in the beginning of Section 2 and $h_{x}(y)=h(y-x)$. The next theorem states that the interacting Heisenberg picture fields are also translation invariant.

Theorem 5. Let $h$ be in $L_{1} \cap L_{2}$. Then for any $x$ in $R^{3}$

$$
U(-x) \varphi(h, t) U(x)=\varphi\left(h_{x}, t\right)
$$

where $U(x)$ is the unitary group of space translations and $h_{x}(y)=h(y-x)$.

Proof. Since $\varphi^{t}(h)$ is translationinvariant, it is enough to prove that $\varphi(h, t)-\varphi^{t}(h)$ is translation invariant. Due to the uniform norm estimate in Theorem 2, it is enough to prove that $\varphi(h, t)-\varphi^{t}(h)$ is translation invariant for $h$ with compact support $S$. By weak convergence and Theorem 3 it is enough to prove that $\varphi_{\varepsilon_{n}, \Lambda, t}\left(h_{x}\right)-\varphi^{t}\left(h_{x}\right)$ and

$$
U(-x)\left(\varphi_{\varepsilon_{n}, A, t}(h)-\varphi^{t}(h)\right) U(x)=\varphi_{\varepsilon_{n}, \Lambda_{x}, t}\left(h_{x}\right)-\varphi^{t}\left(h_{x}\right)
$$

have the same weak limits when $S_{t}$ is contained in $\Lambda$. The identity above follows from the identity

$$
U(-x) V_{\varepsilon, A} U(x)=V_{\varepsilon, A_{x}} .
$$

But that $\varphi_{\varepsilon_{n}, A, t}\left(h_{x}\right)-\varphi^{t}\left(h_{x}\right)$ and $\varphi_{\varepsilon_{n}, A_{x}, t}\left(h_{x}\right)-\varphi^{t}\left(h_{x}\right)$ have the same limit follows immediately from Theorem 3 . This proves the theorem.

Remark. To get rotational invariance of the Heisenberg picture fields, we have only to choose $g(x)$ rotational invariant. Since then all the cutoff fields $\varphi_{\varepsilon, r, t}(h)$ will be rotational invariant. 
Even though the interaction is formally relativistic invariant, we can not at this stage at least prove that the Heisenberg picture fields are relativistic invariant or not. Already for the time translation we do not even know if it is implemented by a group of $C^{*}$-automorphisms or not. So probably stronger results then weak convergence of the cut-off fields would be needed to discuss relativistic invariance.

\section{References}

1. Glimm, J.: Boson fields with nonlinear selfinteraction in two dimensions. Commun. Math. Phys. 8, 12-25 (1968).

2. - Boson fields with the : $\Phi^{4}$ : interaction in three dimensions. Commun. Math. Phys. (to appear).

3. - and A. Jaffé: A Yukawa interaction in infinite volume. To appear.

4. - - A $\lambda \varphi^{4}$ field theory without cutoffs. Physical Review. To appear.

5. Guenin, M.: On the interaction picture. Commun. Math. Phys. 3, 120-132 (1966).

6. Høegh-Krohn, R.: Boson fields under a general class of cut-off interactions. Commun. Math. Phys. 12, 216-225 (1969).

R. Høegh-Krohn Mathematical Institute

University of Oslo

Oslo, Norway 